\title{
A 48-year-old man with a pleural-based consolidation
}

\author{
Massimo Miniati MD PhD
}

A 48-year-old man underwent surgery for bilateral inguinal hernias. His clinical history was unremarkable except for heavy smoking (52 pack-years). After surgery, the patient had persistent pain in his groin that restricted his physical activity for about 40 days. Two months after surgery, he had an episode that involved shortness of breath and chest pain on his right side that resolved spontaneously after a few days. At this time, the patient did not seek medical help. Four months after surgery, the patient had a similar episode, this time coughing up blood.

A radiograph of the patient's chest showed a pleural-based, cushion-like consolidation adjacent to the right costophrenic angle (Figure 1). Pneumonia was suspected, and the patient was given a prescription for a 7-day course of amoxicillin clavulanate.

When a follow-up chest radiograph 3 days after antibiotic therapy ended showed no changes in lung density, thoracic computed tomography (CT) was ordered. The image showed a pleural-based, convex consolidation in the lower lobe of the patient's right lung (Figure 2). No pleural effusion nor hilar or mediastinal lymphoadenopathy was seen. Computed tomography angiography showed no intravascular filling defects. A standard angiography protocol for pulmonary embolism was not used, because pulmonary embolism had not been included in the differential diagnosis. Given the patient's history of smoking, the possibility of malignant disease was considered, and a CTguided needle biopsy of the area of consolidation was done. The specimen showed erythrocytes and hemosiderin-laden phagocytes, but no malignant cells. The patient refused further diagnostic procedures.

Two months after this work-up (6 mo after surgery), the patient noticed a progressive swelling of his right calf and again reported severe shortness of breath and right-sided chest pain. A CT pulmonary angiogram showed multiple filling defects, suggesting pulmonary embolism (Figure 3A). Contrast-enhanced angiography showed no dilatation of the right heart cavities or leftward displacement of the interventricular septum (Figure 3A). A caudal section of the right lung showed the organization of the former consolidation, and a new, pleuralbased, hemispherical consolidation was recognized as pulmonary infarction (Figure 3B). Using compression ultrasonography, right popliteal deep vein thrombosis was diagnosed.

Treatment consisted of a weight-adjusted daily dose of fondaparinux, followed by a 6 month regimen of anticoagulant agents taken orally. No inherited thrombophilia was found on further investigation. Three months later, the patient had recovered clinically, and radiography showed no residual abnormalities of the chest other than blunting of the right costophrenic angle. Results of perfusion lung scintigraphy were normal, showing a preserved base-to-apex gradient of blood flow distribution. On transthoracic echocardiography, the end-diastolic diameter of the patient's right ventricle was normal, as was the thickness of the right ventricular free wall. No tricuspid regurgitation was seen.

\section{Discussion}

Pulmonary embolism is a common but underdiagnosed condition. In a survey of the literature from 1945 to 2002, the condition was unsuspected or undiagnosed antemortem in $84 \%$ of patients in whom it was found at autopsy. ${ }^{1}$ The condition may escape prompt diagnosis because its clinical symptoms and signs are nonspecific. ${ }^{2,3}$

\section{KEY POINTS}

- Pulmonary infarction may occur in young and otherwise healthy patients, thus any pleural-based consolidation (cushion-like or hemispherical) should prompt consideration of such an event as a possibility.

- Pulmonary infarction is not always accompanied by hemoptysis, pleuritic chest pain or a pleural friction rub.

- If a pulmonary consolidation is consistent with infarct, pulmonary embolism has occurred, and the diagnosis should be confirmed promptly.

- Prediction models can guide decision-making during investigation for pulmonary embolism.
Competing interests: Massimo Miniati was involved in the development of several clinical prediction models for pulmonary embolism.

This article has been peer reviewed.

Correspondence to: massimo.miniati@unifi.it

CMAJ 2013. DOI:10.1503 /cmaj.111190 
Pulmonary infarction is an uncommon manifestation of pulmonary embolism.

This patient's case shows how lung densities related to pulmonary infarction from pulmonary embolism may be misinterpreted as evidence of pneumonia or lung cancer. The radiographic appearance of a pulmonary infarct has classically been described as a triangular shadow with the apex pointing toward the heart; however, other patterns may be more common, as will be described later in this report. In addition, the occurrence of sudden, unexplained dyspnea with pleuritic chest pain may have been overlooked owing to the patient's hemoptysis and extensive history of smoking, thus highlighting the need for objective tools for determining the likelihood of pulmonary embolism.

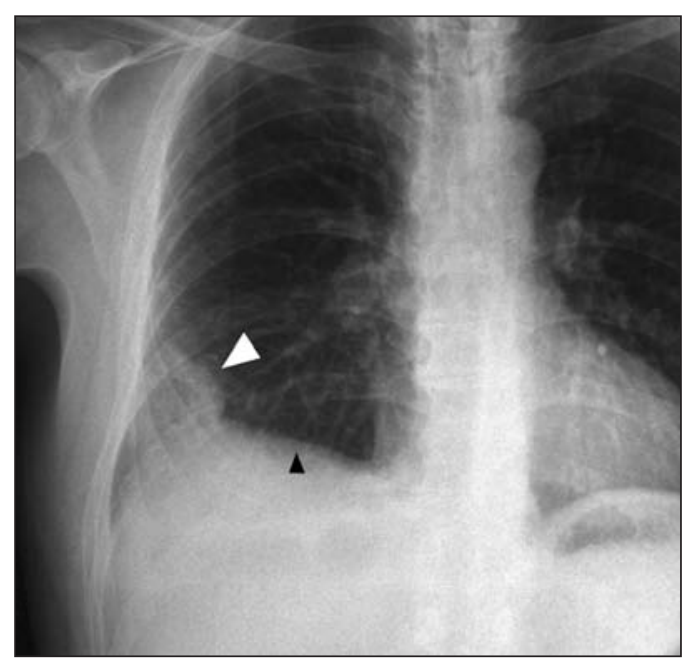

Figure 1: Posteroanterior radiograph of the chest of a 48-year-old man with dyspnea, pain in his right chest and hemoptysis. A pleural-based, cushionlike consolidation adjacent to the right costophrenic angle can be seen (white arrowhead). Slight elevation of the right hemidiaphragm is also evident (black arrowhead).

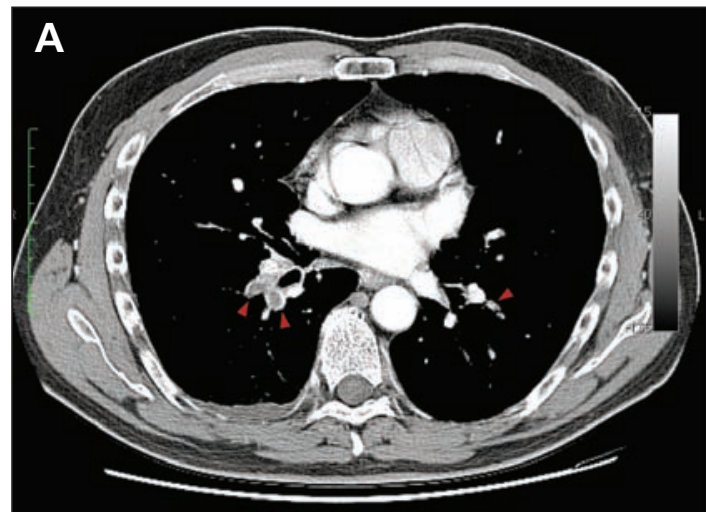

\section{Diagnosing pulmonary embolism}

Abrupt dyspnea is the most frequent symptom of pulmonary embolism, followed by chest pain (usually pleuritic) and syncope. ${ }^{4-6}$ The occurrence of these symptoms, if not explained otherwise, should alert the clinician to consider pulmonary embolism as part of the differential diagnosis and order appropriate objective testing. ${ }^{46}$

There is increasing awareness of the importance of assessing clinical probability in patients suspected of having pulmonary embolism, and prediction rules have been introduced. ${ }^{7-11}$ Based on one such model, ${ }^{9}$ the patient described here

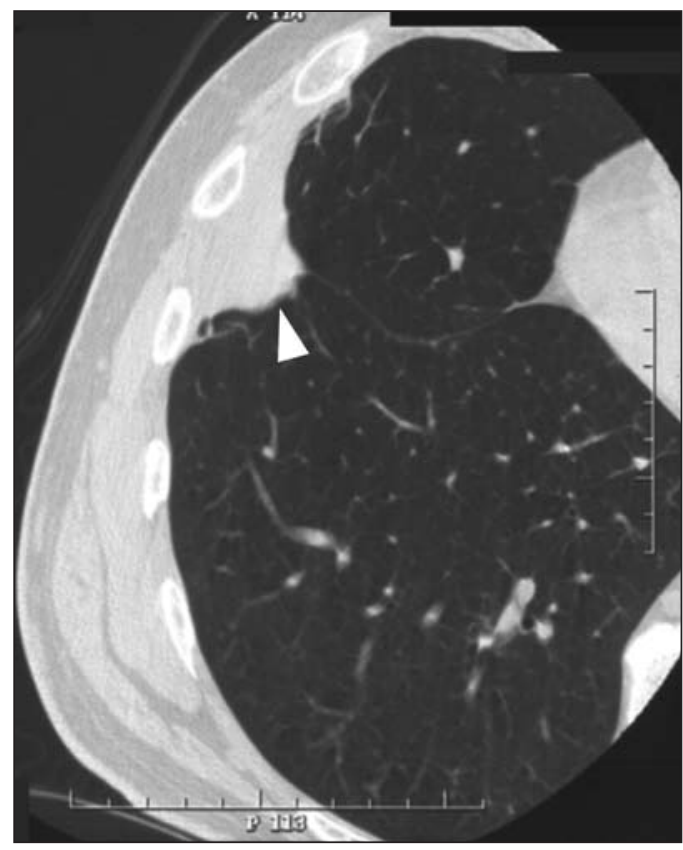

Figure 2: Computed tomography scan of the patient's chest taken 10 days after the radiograph shown in Figure 1, showing a pleural-based, convex consolidation in the right lower lobe (white arrowhead). The shape of the consolidation is consistent with pulmonary infarction.

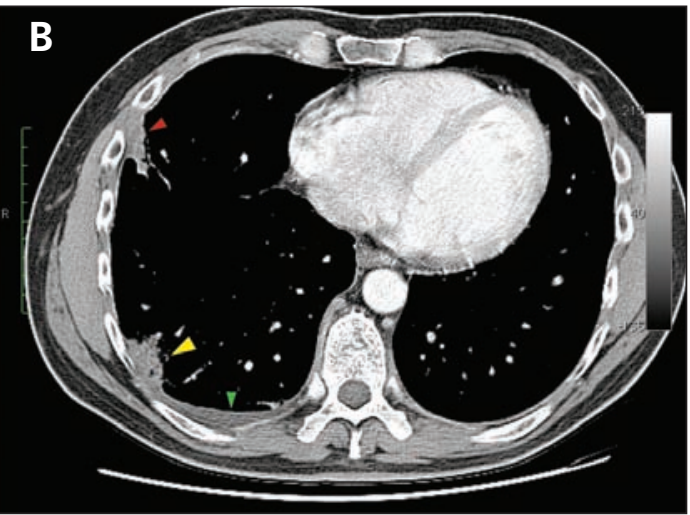

Figure 3: (A) Thoracic computed tomography (CT) scan taken 46 days after the first CT study (Figure 2) showing multiple intravascular filling defects suggestive of pulmonary embolism (red arrowheads). (B) Caudal CT section of the right lung showing the organization of the former consolidation (red arrowhead) and a new pleural-based consolidation consistent with infarction (yellow arrowhead). A small pleural effusion is also present (green arrowhead). 
would have been given a clinical (pretest) probability of $98 \%$ (Box 1). With this pretest probability, the estimated post-test probability of pulmonary embolism, even with a negative angiogram (CT angiography has a sensitivity of $83 \%$ and a specificity of $96 \%$ ), would be as high as $89.7 \%{ }^{12}$ This should mandate further investigation, such as compression ultrasonography of the lower limb or lung scintigraphy.

\section{Diagnosing pulmonary infarction}

Hemoptysis, pleuritic chest pain and pleural friction rub, traditionally regarded as suggestive of pulmonary infarction, have limited predictive value diagnosing the condition. ${ }^{2,3}$ The diagnosis rests on the correct recognition of a lung density on chest radiography. ${ }^{13,14}$

In radiologic-pathologic correlations, Hampton and Castleman ${ }^{13}$ first refuted the idea that pulmonary infarcts appear conical or pyramidal in shape, the apical portion of which is spared from infarction because of sufficient collateral blood supply via the bronchial circulation. Rather, they showed, ${ }^{13}$ as did Fleischner, ${ }^{14}$ that the more common appearance of a pulmonary infarct is of a cushion-like or hemispherical consolidation with the base lying along the surface of the visceral pleura (e.g., the lateral, diaphragmatic, mediastinal or interlobar pleura.) Most infarcts are located in the lower portions of the lungs, fewer in the midportions and even fewer in the upper portions. ${ }^{13,14}$ This is in keeping with the regional distribution of pulmonary blood flow, with the lower lung regions receiving twice as much blood as the upper regions. ${ }^{15}$

Hampton and Castleman also challenged the belief that every pulmonary infarct proceeds through sequential stages of congestion, extravasation, necrosis and healing. ${ }^{13}$ They saw that, in some cases, extravasation of blood into the lung is not followed by alveolar destruction and subsequent scar formation, thus coining the definition of "incomplete and reversible infarction." This condition manifests as an infarct-like consolidation on radiography that vanishes completely in a matter of days.

The introduction of multidetector CT imaging has greatly improved the evaluation of lung densities by providing 3-dimensional image reconstruction. Revel and coworkers ${ }^{16}$ retrospectively examined 150 peripheral lung consolidations on CT imaging from 134 patients, 50 of whom had intravascular filling defects consistent with pulmonary embolism. In this population, the presence of central lucencies within a peripheral consolidation had a $98 \%$ specificity for infarction, but a sensitivity of only $46 \% .{ }^{16}$ Pulmonary infarction is likely if a peripheral consolidation contains central lucencies, but only a small proportion of infarcts have such an abnormality. Although the authors used the term "triangular" to refer to the shape of the infarct, on close examination, the images shown in their paper conform to the description given by Hampton and Castleman - that is, pleural-based and hemispherical in shape. ${ }^{16}$

This patient's case prompted the re-evaluation of chest radiographs and CT images of 212 patients in whom pulmonary embolism had been diagnosed at our institution over a 1-year period as a quality-control exercise. Lung consolidations consistent with infarction, according to Hampton and Castleman's criteria, were identified in $44(20.7 \%)$ of the patients. Patients with infarct were younger than those without infarct. Only 6 patients with infarct (13.6\%) presented with hemoptysis. Notably, infarction had been correctly recognized by the radiologists in 16 patients $(36.4 \%)$, whereas it was mistaken for pneumonia in 23 patients (52.3\%) and for lung cancer in 5 patients $(11.3 \%)$.

Although it is important to recognize that the more common radiological appearance of a pulmonary infarct is of a cushion-like or hemispherical consolidation with the base lying along the surface of the visceral pleura, clinical suspicion of an acute pulmonary event such as embolus or infarct and the use of prediction tools may provide a check to over-reliance on any single radiological sign.

\section{Box 1: Using a prediction model for pulmonary embolism ${ }^{9}$}

There are several prediction models available to guide decision-making around investigation for pulmonary embolism. ${ }^{7-11}$ My colleagues and I have developed a logistic regression model* to predict the clinical (pretest) probability of pulmonary embolism. ${ }^{9}$ This model includes the following variables: age, sex, history of deep vein thrombosis, previous cardiac or pulmonary disorders, clinical symptoms (sudden onset dyspnea, chest pain, fainting or syncope, hemoptysis), high fever $\left(>38^{\circ} \mathrm{C}\right)$, electrocardiographic signs of acute right ventricular overload and chest abnormalities on radiography (oligemia, amputation of the hilar artery, consolidation suggestive of infarction, consolidation other than infarction, pulmonary edema). Each variable is assigned a regression coefficient. The sum of regression coefficients that apply to a particular patient is added to a constant (-3.26). The probability of pulmonary embolism equals $1 /\left[1+\mathrm{e}^{-(\text {sum }+-3.26)}\right]$.

The coefficients that apply to this patient's case are: age less than 63 years $=$ 0 ; male sex $=0.81$; sudden-onset dyspnea $=1.29$; chest pain $=0.64$; hemoptysis $=0.89$; and pulmonary infarction $=3.55$.

Using our equation, the estimated probability can be calculated as follows:

$0+0.81+1.29+0.64+0.89+3.55=7.18$

$7.18+(-3.26)=3.92$

$1 /\left(1+\mathrm{e}^{-3.92}\right)=98 \%$.

Thus, this patient had a $98 \%$ probability of pulmonary embolism.

*An online version to compute the probability of pulmonary embolism is available at www.ifc.cnr.it/pisamodel. The tool can be downloaded for use on mobile phones, tablets and desktop computers. The website also includes a simplified model for users who are not familiar with the interpretation of the chest radiograph. ${ }^{11}$ 


\section{References}

1. Stein PD. Prevalence, risks, and prognosis of pulmonary embolism and deep vein thrombosis. In Pulmonary embolism, 2nd ed. Oxford (UK): Blackwell Publishing; 2007, pp. 3-15.

2. Wenger NK, Stein PD, Willis PW III. Massive acute pulmonary embolism. The deceivingly nonspecific manifestations. JAMA 1972;220:843-4.

3. Bell WR, Simon TL, DeMets DL. The clinical features of submassive and massive pulmonary emboli. Am J Med 1977;62:355-60.

4. Stein PD, Willis PW III, Dalen JE. Importance of clinical assessment in selecting patients for pulmonary arteriography. Am J Cardiol 1979;43:669-71.

5. Miniati M, Prediletto R, Formichi B, et al. Accuracy of clinical assessment in the diagnosis of pulmonary embolism. Am J Respir Crit Care Med 1999; 159:864-71.

6. Miniati M, Cenci C, Monti S, et al. Clinical presentation of acute pulmonary embolism: a survey of 800 cases. PLoS ONE 2012; 7:e30891.

7. Wells PS, Anderson DR, Rodger M, et al. Derivation of a simple model to categorize patients probability of pulmonary embolism: increasing the model utility with the SimpliRED Ddimer. Thromb Haemost 2000;83:416-20.

8. Wicki J, Perneger TV, Junod AF, et al. Assessing clinical probability of pulmonary embolism in the emergency ward: a simple score. Arch Intern Med 2001;161:92-7.

9. Miniati M, Monti S, Bottai M. A structured clinical model for predicting the probability of pulmonary embolism. Am J Med 2003; 114:173-9.
10. Le Gal G, Righini M, Roy PM, et al. Prediction of pulmonary embolism in the emergency department: the revised Geneva score. Ann Intern Med 2006;144:165-71.

11. Miniati M, Bottai M, Monti S, et al. Simple and accurate prediction of the clinical probability of pulmonary embolism. Am J Respir Crit Care Med 2008;178:290-4.

12. Stein PD, Fowler SE, Goodman LR, et al. Multidetector computed tomography for acute pulmonary embolism. N Engl J Med 2006; 354:2317-27.

13. Hampton AO, Castleman B. Correlation of postmortem chest teleroentgenograms with autopsy findings with special reference to pulmonary embolism and infarction. AJR Am J Roentgenol 1940;43:305-26.

14. Fleischner FG. Roentgenology of pulmonary infarct. Semin Roentgenol 1967;2:61-76.

15. Giuntini C, Mariani M, Barsotti A, et al. Factors affecting regional pulmonary blood flow in left heart valvular disease. Am J Med 1974;57:421-36.

16. Revel MP, Triki R, Chatellier G, et al. Is it possible to recognize pulmonary infarction on multisection CT images? Radiology 2007; $244: 875-82$

Affiliation: Dipartimento di Medicina, Sperimentale e Clinica, Università di Firenze, Firenze, Italy

Acknowledgement: Logistic regression analysis was carried out by Matteo Bottai, Unit of Biostatistics, Karolinska Institutet, Stockholm, Sweden.

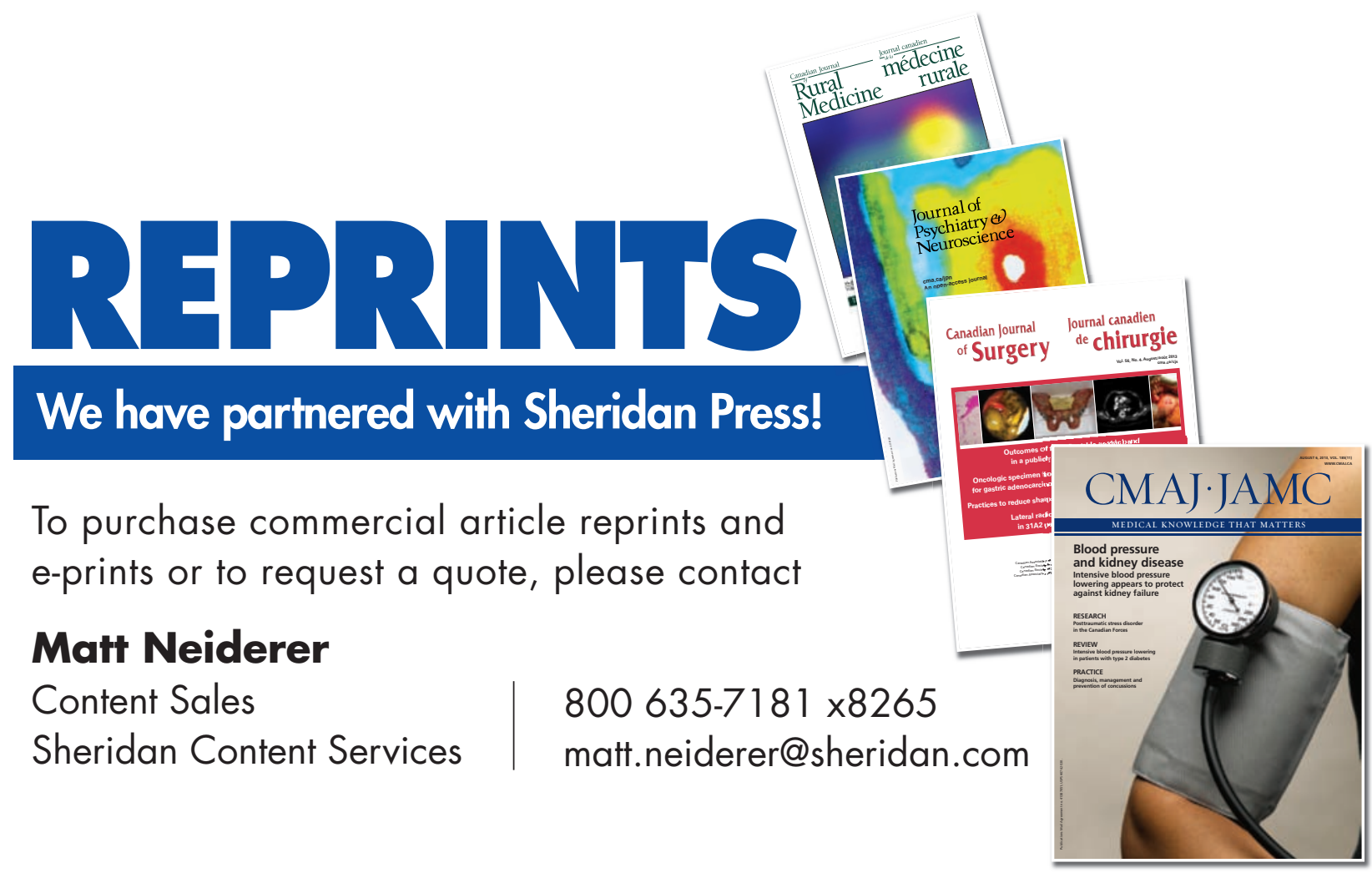

